\title{
Temperature Effect on Pump Oil and Alkanes Evaporation
}

\author{
Nathaniel A. Waldstein and Alex A. Volinsky \\ Department of Mechanical Engineering, University of South Florida, Tampa FL \\ 33620, U.S.A., volinsky@eng.usf.edu
}

\begin{abstract}
There are many products, including hard drives, which require trace amounts, on the order of several milligrams, of lubricants for proper operation. The following study investigated the evaporation rates of pump oil and several alkanes, which have a wide range of applications. Both static and dynamic temperature tests were conducted. The rate of evaporation of the test specimen was determined as the mass loss per unit time. Using the Arrhenius equation, the activation energy of the evaporation process, $E_{a}$, can be calculated as the slope of the best fit line for a plot of the $\ln (\mathrm{k})$ vs. 1/T (where $\mathrm{k}$ represents the evaporation rate). The alkanes were compared using the activation energy required for evaporation as model systems. Pump oil was also evaporated from two types of microchannels. Developed testing procedures help speed up new lubricant formulations qualification.
\end{abstract}

\section{INTRODUCTION}

Great advancements in storage capacities and reading/writing speeds have pushed the hard disk drive materials to their limits. Modern hard drives can operate at 10,000 RPM and greater, and these speeds have generated taxing demands on the life of a hard drive. These elevated rotational speeds inherently cause the hard drives to operate at higher temperatures than in the past. The greater the operating temperature the faster the lubricating oil in the hard drive motor will evaporate inevitably resulting in hard drive failure over time. Current industry conventions require the testing of new lubricants over a period of several months. This hinders the development of new lubricant formulas and ultimately delays the release of superior hard drives to the market. This study provides a methodology that will reduce this development stage from months to days. Samples were tested utilizing thermogravimetrics and analyzed with the Arrhenius equation.

The Swedish born scientist, Svante Arrhenius (1859-1927), developed what is now known as the Arrhenius equation [1]. Arrhenius noted that the majority of chemical reactions need additional energy to continue. This energy, specifically heat energy, is added to a system until a predetermined threshold is reached and the reaction commences. This threshold is a concept that was developed by Arrhenius and is referred to as the activation energy. Arrhenius further developed these concepts and combined supportive ideas to formulate the Arrhenius equation. Simply, this equation relates the activation energy to the rate of the reaction process. Specifically, it was derived to adequately account for the temperature effects on the reaction 
rates of gases $[1,2]$. The Arrhenius equation was originally derived from the work of the Dutch chemist Jacobus Henricus van 't Hoff (1852 - 1911) [1]. In order for Arrhenius to explain simple chemical reactions, he viewed most processes as simple $1^{\text {st }}$ order reactions that have distinct temperature characteristics and obvious activation energies. Even though evaporation is a physical process and not a chemical one, this approach is still valid. These reaction fundamentals are obtained by plotting the natural logarithm of the reaction rate against the inverse of the absolute temperature, which provides a model that relates the reaction rate to temperature. One form of the Arrhenius Equation is an integration of the underlying differential equation and is presented in the following empirical expression:

$$
k=A e^{\left(-\frac{E_{a}}{R T}\right)}
$$

where $k$ is a constant that correlates to the rate of the reaction, $E_{a}$ is the activation energy of the reaction $(\mathrm{J} / \mathrm{mol}), T$ is the absolute temperature, $R$ is the universal gas constant $(8.314472$ $\mathrm{J} /(\mathrm{K} \cdot \mathrm{mol}))$, and $A$ is the pre-exponential constant, which has the same units as $k$. The units depend on the order of the reaction. For an $n^{\text {th }}$ order reaction, the shared units are $\left(\mathrm{mol}^{1-\mathrm{n}} \mathrm{L}^{\mathrm{n}-1}\right.$ /sec), however, in this investigation the rate of evaporation was measured experimentally and has the units of mass per unit time as does the pre-exponential constant $A$. Since the activation energy appears in a nonlinear form in equation (1), problems arise during nonlinear regression. As a result, the natural logarithm of both sides of the equation is taken to yield:

$$
\ln k=\ln A-\frac{E_{a}}{R T}
$$

If the activation energy, $E_{a}$, and the pre-exponential constant, $A$, are not changing with temperature, then a plot of $\ln (k)$ against the inverse of $T$ will result in a straight line whose slope is proportional to the activation energy and offset is the natural logarithm of the pre-exponential constant [3]. This can be seen in Figure 1. In this example, the slope of the linear best fit line multiplied by the negative of the universal gas constant provides an estimate of the activation energy of the evaporation. In this case, the activation energy of the evaporation of undecane is $50.4 \mathrm{~kJ} / \mathrm{mol}$. Likewise, taking the exponential of the offset of the best fit line provides a value of the pre-exponential constant to be $0.27 \mathrm{mg} / \mathrm{sec}$. If the plot is not linear as previously described, then the activation energy changes with temperature $[1,2]$. 


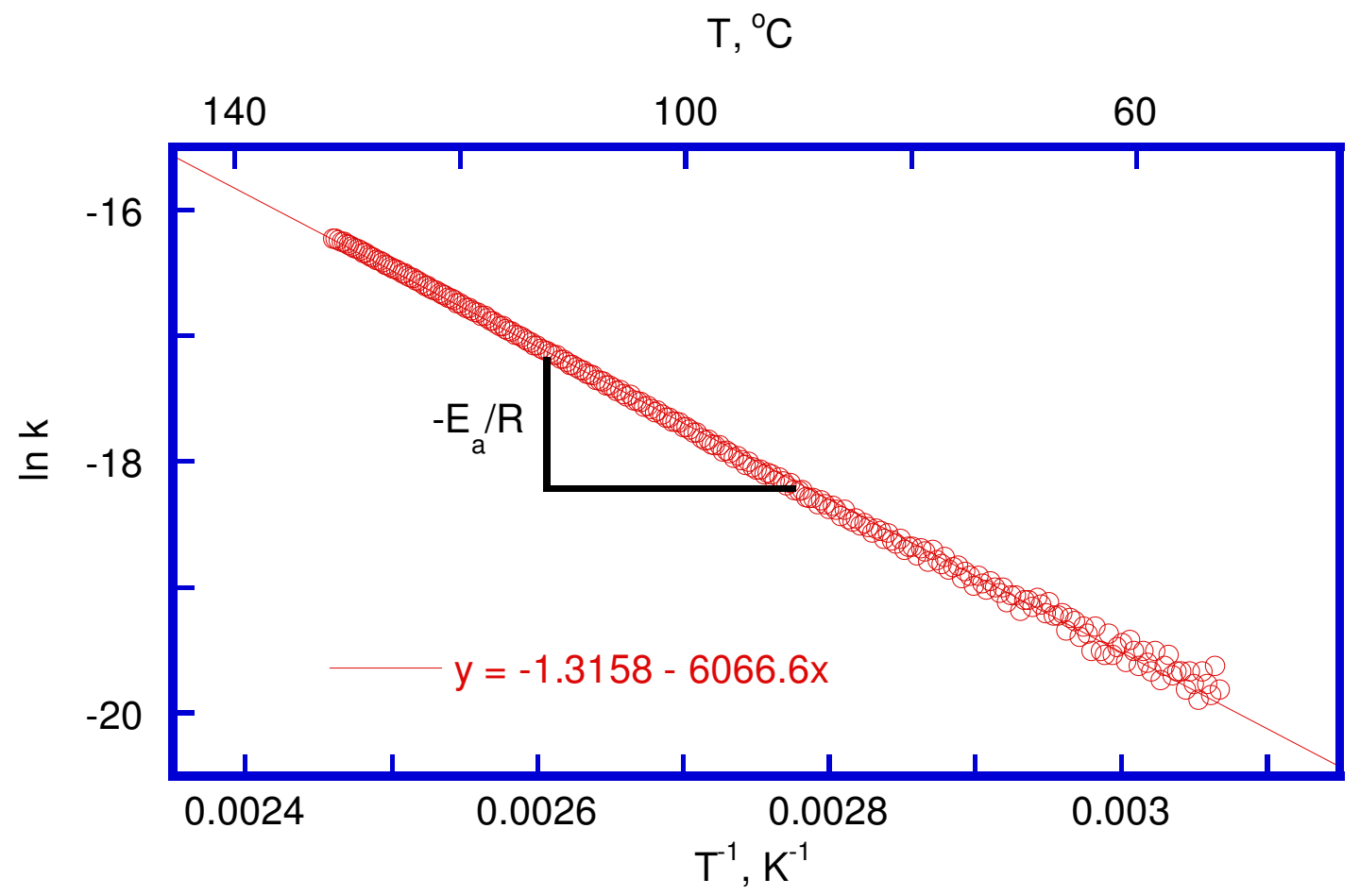

Figure 1. A typical Arrhenius plot for the calculation of the activation energy, $E_{a}$, for the evaporation of undecane.

\section{EXPERIMENT}

All testing followed the same procedure and used the same testing equipment. Samples were taken from storage containers using a micropipette, $9 \mathrm{mg}$ for alkane testing and 20-25 mg for pump oil tests. The micropipette was used to inject the samples into aluminum pans and the pans were then placed into a thermogravimetric apparatus. The pan with the sample was balanced with an empty identical aluminum pan and the microbalance was zeroed out. The particular microbalance used has a resolution of $0.001 \mathrm{mg}$, a range of $\pm 500 \mathrm{mg}$, and a maximum gross sample weight limit of $1 \mathrm{~g}$. The pans were then heated inside a heating chamber with a programmable temperature profile and the differential temperatures were recorded. The temperatures of the empty pan and the pan that contained the sample substance were measured simultaneously, as well as the temperature inside the heating chamber. The chamber was connected to a passive exhaust and the inside temperature was used as a baseline temperature. The difference in temperature of the two pans was then used to accurately determine the temperature of the sample to within $0.1^{\circ} \mathrm{C}$. The temperature and the weight of the sample were measured continuously throughout testing runs. The experiments were concluded when the sample was completely evaporated or until the heating program ended. The equipment used during testing was highly accurate and proved to be a very good comparative tool for the evaporation of different substances [3-8]. 


\section{RESULTS AND DISCUSSION}

Testing of this study was conducted in both static and dynamic regimes. Static testing was when a sample was held isothermal for an extended period of time before the temperature was either increased or decreased. Conversely, during dynamic testing the temperature of the sample was continuously increased. As a result, static testing was a much longer process than the dynamic testing. Dynamic testing proved to be more valid for determining the activation energy of a sample at higher temperatures, while static testing was more appropriate at lower temperatures $\left(30-75^{\circ} \mathrm{C}\right)$. As expected, static testing resulted in greater values of activation energies. Combining the data retrieved from both types of testing and graphing them on the same Arrhenius plot provides a more complete profile of the relationship of the activation energy to that of temperature. Figure 2 shows an example of combined static and dynamic profiles for hexadecane.

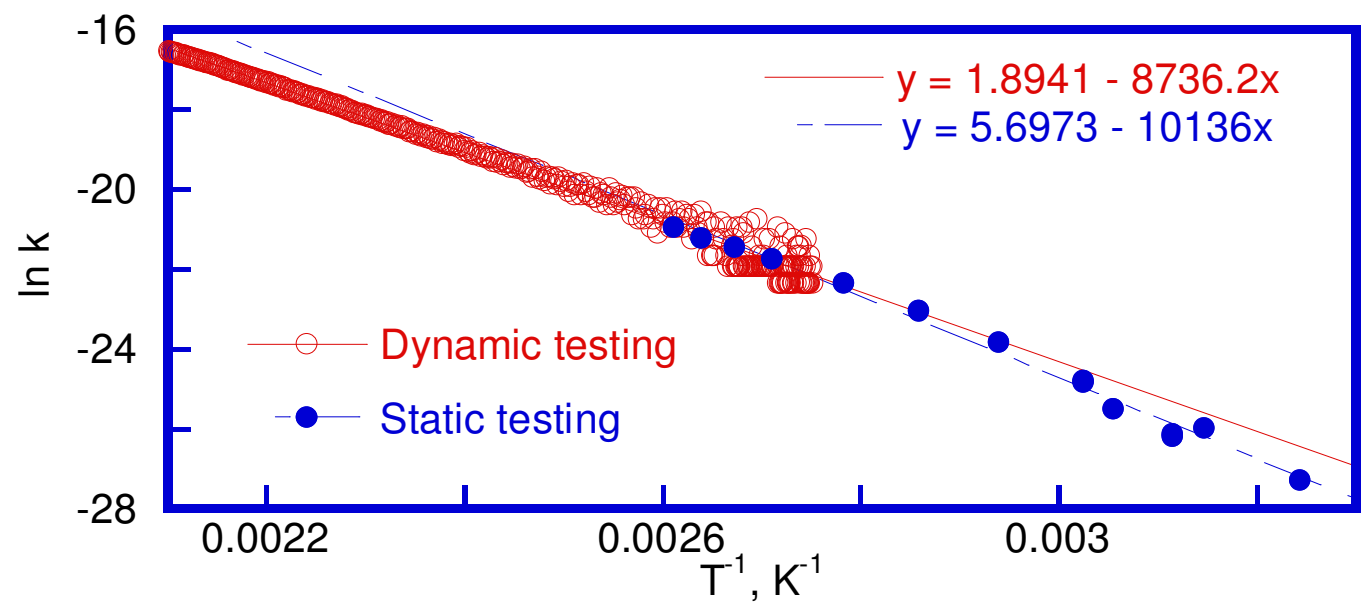

Figure 2. Static and dynamic Arrhenius plots for hexadecane.

Table 1. Activation energies for alkanes, obtained from static and dynamic testing.

\begin{tabular}{|c|c|c|}
\hline \multirow{2}{*}{ Alkanes } & Static & Dynamic \\
\cline { 2 - 3 } & \multicolumn{2}{|c|}{$E_{a}, \mathrm{~kJ} / \mathrm{mol}$} \\
\hline Undecane & $79.5 \pm 0.11$ & $52.5 \pm 0.13$ \\
\hline Dodecane & $74.5 \pm 0.11$ & $54.8 \pm 0.15$ \\
\hline Tridecane & $72.2 \pm 0.12$ & $54.9 \pm 0.16$ \\
\hline Tetradecane & $85.4 \pm 0.11$ & $53.6 \pm 0.16$ \\
\hline Pentadecane & $89.3 \pm 0.12$ & $70.9 \pm 0.15$ \\
\hline Hexadecane & $82.9 \pm 0.13$ & $70.4 \pm 0.18$ \\
\hline
\end{tabular}

Table 1 lists the activation energies for the alkanes evaporation retrieved from static and dynamic testing. In static testing the sample weight was monitored at constant temperature 
$\left(0.001{ }^{\circ} \mathrm{C} / \mathrm{min}\right.$ variation) for hours, after which the temperature was changed $\left(30-120{ }^{\circ} \mathrm{C}\right.$ range $)$ and the test repeated. During dynamic testing the sample temperature was increased continuously from room temperature to $300{ }^{\circ} \mathrm{C}$ at a rate of $30{ }^{\circ} \mathrm{C} / \mathrm{min}$. Due to such a high chamber temperature, as compared to the boiling point of the alkanes, all of the samples completely evaporated prior to achieving this temperature. From Table 1 it can be seen that the reported activation energy is significantly higher for static vs. dynamic tests. This is expected because the slower temperature increase results in the slower evaporation process. Additionally, a sample held isothermal at lower temperatures requires a greater amount of energy needed for molecules to break free from the surface of the evaporating liquid.

In addition to the alkanes, turbo-molecular pump (TMP) oil was also evaporated. The oil was tested with similar testing procedures, but the samples were contained in different fixtures. As opposed to aluminum pans the TMP oil was tested in microchannels that simulate the microchannels in many consumer products, including hard drives. In these experiments the oil was constricted to the threads of a bolt and a nut, both made of aluminum. This effectively mimics the movement of the evaporating liquid through microchannels and allows for evaporation only at the point of air exposure. A similar fixture was made without threads, which resulted in data similar to that of the TMP oil evaporating from an aluminum pan. Figure 3 shows an Arrhenius plot and the evaporation rates for these two fixtures. As expected, greater activation energy is required for TMP oil evaporation from a microchannel and the evaporation rate is much slower.
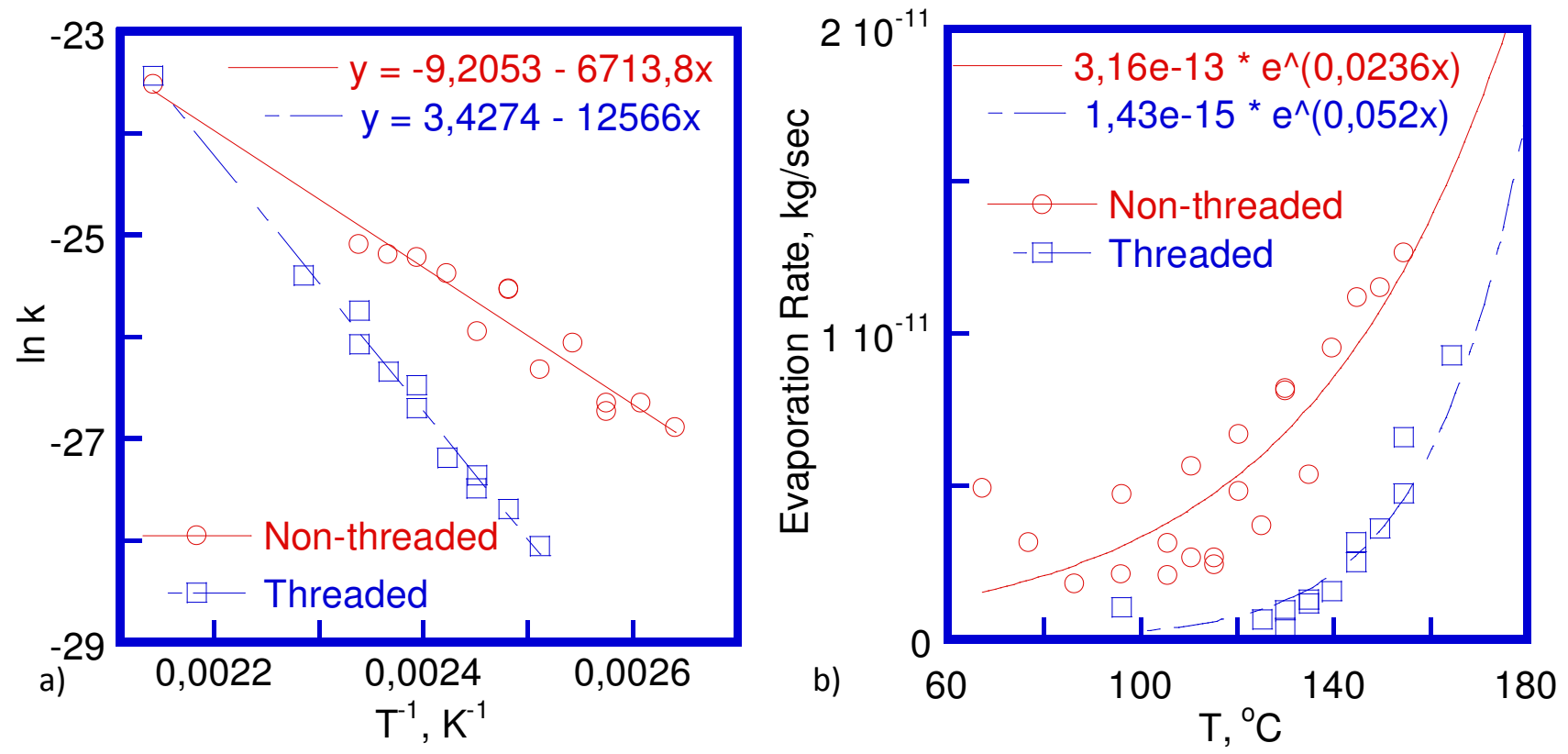

Figure 3. a) Arrhenius plot and b) evaporation rates of TMP oil for threaded and non-threaded fixtures. 


\section{CONCLUSIONS}

The evaporation characteristics have been investigated for alkanes and pump oil. Understanding how a substance will evaporate can be very useful for comparative purposes and selection of the best lubricant for particular applications. It is possible to measure both the evaporation rate and the activation energy directly. Either of these quantitative results can be used as a comparative tool between different substances. The results of dynamic testing produce lower and more consistent calculations of the activation energies of the alkanes. The factors that contribute most to variation of the calculation of these results are the exposed surface area, the heating rate, the flow rate of air, and of course the substance properties themselves such as density, molecular weight and boiling point. It was observed that a mixture of equal proportions of alkanes did not consistently result in the averaging of the evaporation rates. This is because the change in composition is not directly proportional to the change in the entropy of the liquid mixture. The testing helped to prove the theory that in a mixture of alkanes the lighter substance will evaporate first resulting in calculations close to a pure sample of the lighter substance. Future investigations should be conducted to gain a further understanding of the importance of the roles that different factors have on evaporation. From this added information modeling of evaporation is possible and could provide a quick comparative tool of the evaporation of new lubricants.

\section{ACKNOWLEDGEMENTS}

The authors would like to thank Dr. Dirk C. Meyer and his group members at Technical University of Dresden for use of their equipment and samples. Support for this research was provided by NSF under an International Research Education in Engineering (IREE) supplement to the CMMI-600266 grant.

\section{REFERENCES}

1. F.C. Neidhardt, J.L. Ingrahm, M. Schaechter, "Physiology of the Bacterial Cell”, Sinauer Associates Inc. (1990)

2. T.A. McMeekin, J. Olley, T. Ross, D.A. Ratkowsky, "Predictive Microbiology: Theory and Application", Innovation in Microbiology, No. 5, John Wiley \& Sons (1993)

3. P. Aggarwal, D. Dollimore, K. Alexander, Journal of Thermal Analysis Vol. 49, pp. 595-599 (1997)

4. A. A. Mills, J. D. Fry, Eur. J. Phys. Vol. 3, pp. 152-154 (1982)

5. S. F. Wright, P. Phang, D. Dollimore, K. S. Alexander, Thermochimica Acta, Vol. 392393, pp. 251-257 (2002)

6. Y. Cheng, Y. Huang, K. Alexander, D. Dollimore, Vol. 367-368, pp. 23-28 (2001)

7. S. F. Wright, K. A. Alexander, D. Dollimore, Thermochimica Acta, Vol. 367-368, pp. 29-35 (2001)

8. L. Shen, K. S. Alexander, D. Dollimore, Vol. 367-368, pp. 69-74 (2001) 